\title{
Posterior cavoplasty: a new approach to avoid venous outflow obstruction and symptoms for small-for-size syndrome in right lobe living donor liver transplantation
}

\author{
Armin D Goralczyk • Aiman Obed • Alexander Beham • \\ Tung Yu Tsui • Thomas Lorf
}

Received: 22 July 2010 / Accepted: 17 December 2010 /Published online: 5 January 2011

(C) The Author(s) 2010. This article is published with open access at Springerlink.com

\begin{abstract}
Purpose A common and serious problem after living donor liver transplantation (LDLT) of small grafts is small-for-size syndrome (SFSS). Although hyperdynamic portal inflow and portal hypertension are cornerstones in the development of SFSS, inadequate outflow may aggravate SFSS. Therefore, enlargement of the portal outflow tract by incision of the anterior rim of the orifice of the right hepatic vein (RHV) has been advocated for right lobe LDLT. But backwards tilt of a small graft into a large abdominal cavity may lead to a choking of the otherwise large anastomosis and thus we propose posterior enlargement of the orifice of the RHV.

Method In this test-of-concept study, we evaluated portal vein pressure (PVP), clinical parameters, and laboratory
\end{abstract}

Armin D Goralczyk and Aiman Obed contributed equally in this study.

A. D Goralczyk · A. Obed · A. Beham · T. Lorf

Department of General and Visceral Surgery,

University Medical Center Göttingen,

Robert-Koch-Str. 40,

37075 Göttingen, Germany

T. Y. Tsui

Department of Hepatobiliary and Transplant Surgery,

University Medical Center Hamburg-Eppendorf,

Martinistraße 52,

20246 Hamburg, Germany

A. D Goralczyk $(\bowtie)$

University Medical Center Göttingen,

Robert-Koch-Str. 40,

37099 Göttingen, Germany

e-mail: agoralczyk@med.uni-goettingen.de measurements in 22 patients that underwent right lobe LDLT and either received standard end-to-end anastomosis of the RHV or posterior cavoplasty.

Results In patients that underwent posterior cavoplasty, we observed significantly lower PVP and less hyperbilirubinemia. There was a non-significant trend to lower incidence of SFSS. Other laboratory measurements and clinical parameters were not significantly different.

Conclusion We recommend posterior cavoplasty for enlargement of the hepatic venous outflow tract in right lobe LDLT as a method to avoid portal hypertension, hyperbilirubinemia, and possibly SFSS, especially in patients that receive small grafts.

Keywords Liver transplantation · Complication · Surgical technique - Clinical trial · Portal hypertension · Living donor liver transplantation
Abbreviations
LDLT Living donor liver transplantation
SFSS Small-for-size syndrome
RHV Right hepatic vein
PVP Portal vein pressure
IVC Inferior vena cava
IQR Interquartile range
ALT Alanine transaminase
AST Aspartate transaminase
GGT Gamma-glutamyl transferase
PT Prothrombin time
POD Postoperative day
LME Linear mixed effects model
CI Confidence interval
CT Computed tomography 


\section{Introduction}

Small-for-size syndrome (SFSS) injury is a major concern after living donor liver transplantation (LDLT) [1]. It manifests as coagulopathy, encephalopathy, intractable ascites, and prolonged jaundice [2]. SFSS may cause prolonged hospitalization and graft loss, and thus, survival rates of patients with small-for-size grafts are worse than those with adequate graft size [3].

SFSS is frequently encountered in small grafts, i.e., grafts with a graft weight- ratio below $0.8 \%$, but may also be found in recipients of larger grafts $[1,4]$. The initial mechanism of injury seems to be a hyperdynamic portal flow through a small liver graft, leading to excessive shear stress injury of sinusoidal cells [5]. Consequently, early attempts to avert SFSS in small grafts focused on the diversion or reduction of portal inflow, such as splenic artery ligation [6], splenectomy [7, 8], mesocaval shunt [9], or partial portocaval shunt $[8,10,11]$. But Fan and colleagues also suggest that a pivotal factor may also be a venous outflow of the graft, because "in the absence of uniform and adequate outflow, the inflow impedance, graft hypoperfusion, and therefore, portal hypertension are aggravated" [1]. Hence, in patients with outflow obstruction, portal hypertension rather reflects outflow obstruction and is not the cause of graft injury. It follows that in this situation, it may even be deleterious to mitigate portal pressure because it may reduce adequate portal flow that is also necessary for graft regeneration $[12,13]$. Additionally,
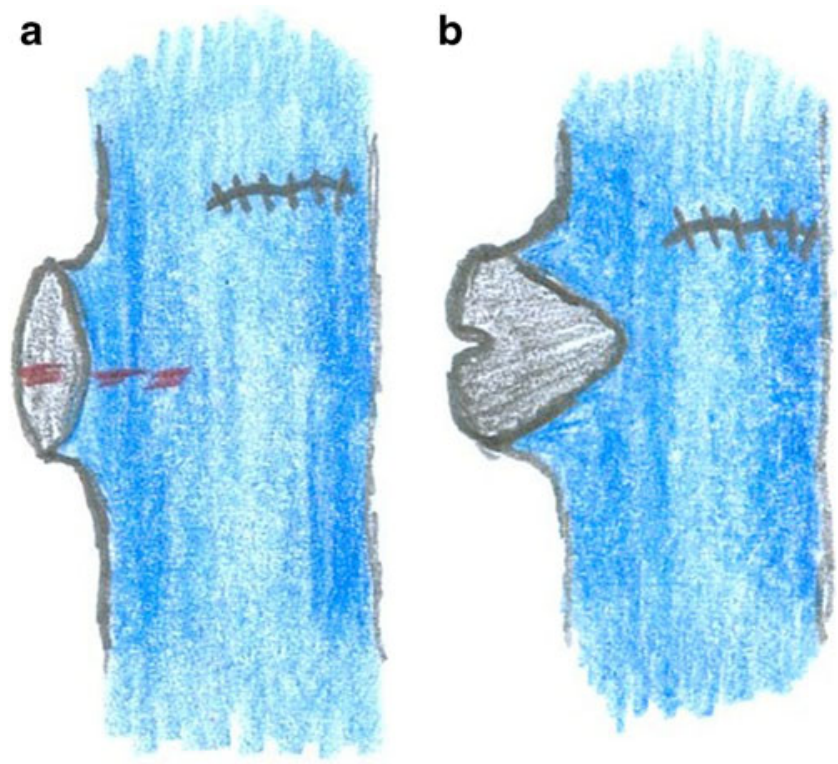

Fig. 1 Anterior cavoplasty (adapted from [1]). a The transverse incision line at the anterior sector of the orifice of the right hepatic vein (RHV) is marked in red. b Orifice has been incised and trimmed to create an anterior enlargement of the venous outflow tract

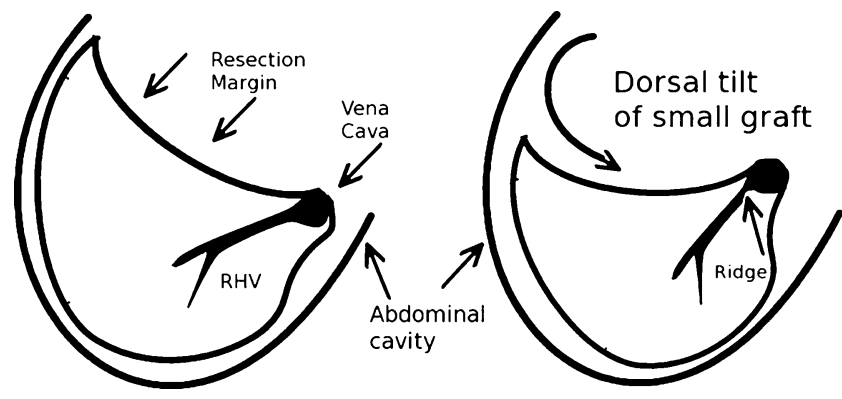

Fig. 2 In case of donor-recipient size mismatch, the small graft may tilt dorsolateral into the large abdominal cavity, and a ridge may develop at the posterior wall of the orifice of the RHV

Boillot, who was one of the first to describe and widely adapt portal decompression to avoid SFSS, has retracted from advocating it in the general situation, because it may cause insufficient portal inflow and subsequent graft failure [14].

To address possible outflow obstruction in right liver grafts, Fan and colleagues [1] advocate anterior incision of the inferior vena cava (IVC) to enlarge the orifice of the right hepatic vein (RHV) and prevent outflow obstruction of the RHV (Fig. 1). But small grafts may also tilt laterodorsal into a large abdominal cavity, and this may lead to functional stenosis by choking on the outflow tract (Fig. 2). Therefore, we enlarged the orifice of the RHV by oval excision of the posterior sector of the orifice (Fig. 3) and compared our new technique of posterior cavoplasty to

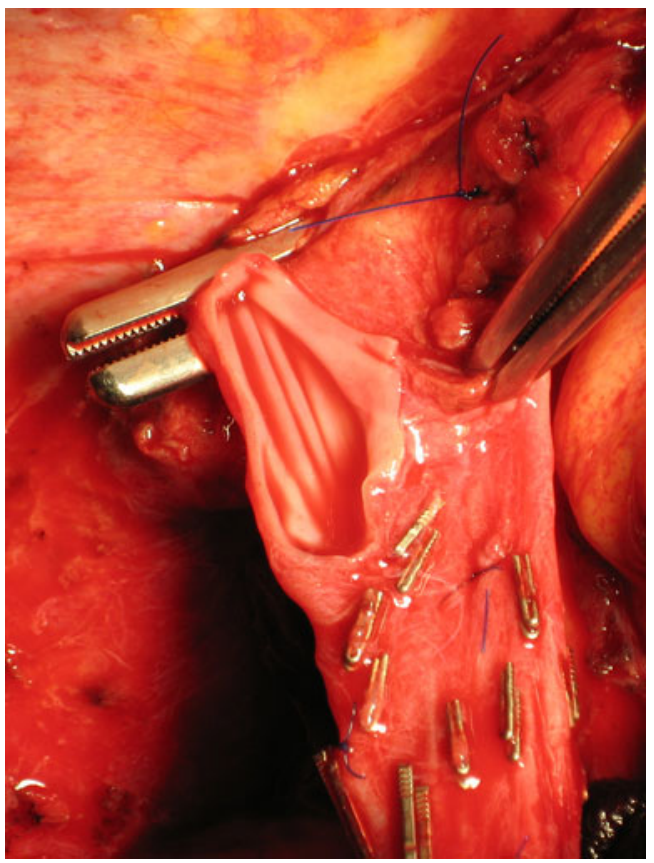

Fig. 3 Posterior cavoplasty by oval excision of the posterior sector of the orifice of the RHV. The anterior rim of the orifice of the MHV is left and also used for the anastomosis to avoid tension 
conventional end-to-end anastomosis of the RHV in a testof-concept study.

\section{Methods}

The study included 22 adult patients who underwent LDLT of the right hepatic lobe between March 2000 and May 2007 , of which seven patients were allocated to the control group and 15 patients to the experimental group that received the new posterior cavoplasty. The first 14 patients underwent systematic allocation by alternate assignment to one of the two study groups in the sequence of their transplantation (pseudo-randomization). After the first interim analysis, there were strong indicators that patients receiving posterior cavoplasty had better clinical outcome, and we decided to allocate all further patients to the experimental group for ethical reasons. Sample size calculations showed that 1:2 allocations would not significantly increase the type II error while retaining the type I error at the predefined level.

The patients ( 6 females and 16 males) had a median age of 46 (interquartile range (IQR) 41-54), a median body mass index of $24.7 \mathrm{~kg} / \mathrm{m} 2$ (IQR 22.2-26.5), and a median graft-to-recipient weight ratio (percentage of recipient body weight) of $0.94 \%$ (IQR $0.83-1.1$ ). Indication for liver transplantation included primary cancer of the liver (six patients), alcoholic liver disease (five patients), hepatitis B or $\mathrm{C}$ virus-related cirrhosis (five patients), cryptogenic cirrhosis (three patients), primary biliary cirrhosis (two patients), and acute-on-chronic autoimmune hepatitis (one patient). Most patients were severely ill, but some were transplanted primarily due to malignancy without reduced liver function (11 patients Child-Pugh class C, 3 patients class $\mathrm{B}$, and 8 patients class $\mathrm{A}$ ).

Portal pressure was measured after exposure of the hepatoduodenal ligament by insertion of a 22-gauge needle into the portal vein. PVP and central vein pressure (CVP) were measured at the same time in millimeter of mercury (mmHg). After LDLT, PVP, and CVP were measured again in the same fashion just before abdominal closure. Graft function was assessed by daily measurements of total bilirubin, alanine (ALT), and aspartate transaminase (AST), gamma-glutamyl transferase (GGT), and prothrombin time $(\mathrm{PT})$ in a standardized fashion. Measurements were taken from $24 \mathrm{~h}$ prior to LDLT until one of the following endpoints was reached: end of the predefined study time (20 days), discharge from hospital, retransplantation, or death of the patient. For diagnosis of SFSS, the following criteria were used: prolonged hyperbilirubinemia (above $10 \mathrm{mg} / \mathrm{dl}$ at POD 14), no signs for biliary duct obstruction and no proof of rejection, therapy refractory ascites, coagulopathy (INR $>1.5)$.
Surgery

In the patients allocated to the control group, the anastomosis of the graft hepatic vein was performed in an end-toend fashion to the stump of the RHV of the recipient. In the experimental group, the posterior wall of the orifice of the RHV was excised $5 \mathrm{~mm}$ in transverse and longitudinal axis, resulting in a laterodorsal position of the caval anastomosis (Fig. 3). Note also that the anterior sector of the cuff of the RHV is not trimmed completely to allow for hypertrophy of the graft without tension at the anastomosis. The RHV is anastomosed to the orifice with a running 5-0 vascular suture. Venoplasty of the grafts RHV was not necessary as all anastomoses could be sewn without tension. In the experimental group, only tributaries to the middle hepatic vein larger than 5-mm diameter were reconstructed, whereas, also smaller tributaries were reconstructed in the control group to avoid congestion of the right paramedian sector [15]. The recipient middle and left hepatic veins were oversewn. The portal vein was anastomosed in typical endto-end fashion, and the branch patch technique was used for anastomosis of the hepatic artery whenever possible. In all patients, an end-to-end anastomosis of the common bile duct was created.

\section{Immunosuppression}

At the time of transplantation, all patients received induction immunosuppression with 500-mg methylprednisolone and 20-mg basiliximab, the latter was repeated on the fourth postoperative day (POD). Immunosuppression was maintained with oral mycophenolat mofetil from the first POD at $1 \mathrm{~g}$ every $12 \mathrm{~h}$ and oral prednisolone with an initial daily dose of $75 \mathrm{mg}$ per day. The latter was tapered by $5 \mathrm{mg}$ and later $2.5 \mathrm{mg}$ every 2 days to a maintenance dose of $7.5 \mathrm{mg}$ per day. On the fifth POD, oral tacrolimus was added to the immunosuppressive regimen starting at $0.5 \mathrm{mg}$ on the first day and increasing daily dose by $1-2 \mathrm{mg}$ to achieve trough plasma level of 4-10 $\mu \mathrm{g}$ per liter.

\section{Statistical analysis}

All statistical calculations were carried out in the $\mathrm{R}$ language and environment for statistical computing (version 2.11.0) with type I error fixed at 0.05. The clinical characteristics of the two groups were represented as frequencies and percentages for categorial variables and median and interquartile range (IQR) for continuous variables. For univariate statistical inference, we used Fisher's exact test for categorial variables [16] and the non-parametric Mann-Whitney $U$ test for continuous variables [17]. For multivariate analysis, we used ordinary least squares method or linear mixed effects models (LME) 
[18] were appropriate. Package "Ime4" (version 0.99937533) available in $\mathrm{R}$ was used to fit LME. Package "multcomp" (version 1.1-7) was used to obtain familywise confidence intervals for the final model preserving the overall type I error for multiple comparisons [19]. Daily laboratory measurements were averaged if there was more than one measurement per day. Missing values were substituted by last observation carried forward. For display of daily mean within groups, a bootstrapped non-parametric 95\% confidence interval is provided [20]. Laboratory measurements at day 20 were also compared by MannWhitney $U$ test.

Kaplan-Meier method was used to estimate overall patient and graft survival. Differences between groups were analyzed by log-rank test.

\section{Results}

Overall median follow-up was 25.5 months (IQR 12.574.75). Both groups were comparable in terms of age, sex, body mass index, Child-Pugh score, and diagnosis (Table 1). Preoperative transaminases were significantly higher in the experimental group, whereas prothrombin time and total bilirubin were not different. Patients in the experimental group had shorter, warm ischemia time but were not different concerning cold ischemia time, anhepatic time, surgery time, and graft weight ratio.

Portal vein pressure

Before hepatectomy, recipients had a median PVP of $14.5 \mathrm{mmHg}$ (IQR 13.25-16), and there was no significant differences between experimental and control group ( $p$ value $=0.98$; see Fig. $4 \mathrm{a}$ ). After implantation of the graft, there is a significant difference of PVP between both groups ( $p$ value $<0.001$ ), although CVP is not different ( $p=$ 0.43). If we subtract CVP from PVP, we can still see a significant correlation between treatment group and PVP ( $p$ value $=0.004)$. Concerning the preoperative PVP, we can see an increase of $3.6 \mathrm{mmHg}$ ( $p$ value $<0.001$, CI 1.7-5.4) in the control group, whereas PVP decreases in the experimental group by $4.9 \mathrm{mmHg}$ ( $p$ value $<0.001$, CI -7.2 to -2.6 ; Fig. $4 b$ ).

\section{Laboratory measurements}

Daily measurements of liver function tests show significantly lower bilirubinemia in patients that did receive posterior cavoplasty ( $p$ value $=0.01$ in LME model). AST
Table 1 Baseline and perioperative characteristics
Continuous variables are given in median (interquartile range); discrete variables are given in count (percent in group).

$P$ values were derived from test statistics of Mann-Whitney

$U$ test or Fisher's exact test for continuous and discrete variables, respectively

\begin{tabular}{|c|c|c|c|}
\hline Group & Control $(N=7)$ & Experimental $(N=15)$ & $P$ value \\
\hline \multicolumn{4}{|l|}{ Demographic features } \\
\hline Age (yr) & $52(43.5-55)$ & $45(38.5-53)$ & 0.3 \\
\hline Female sex & $1(14)$ & $5(33)$ & 0.6 \\
\hline Body mass index $(\mathrm{kg} / \mathrm{m} 2)$ & $25.5(22.1-26.6)$ & $24.6(22.75-25.95)$ & 0.8 \\
\hline Diagnosis: & & & 0.8 \\
\hline - Cancer & $3(43)$ & $3(20)$ & \\
\hline - Alcoholic liver disease & $1(14)$ & $4(27)$ & \\
\hline - Chronic viral hepatitis & $1(14)$ & $4(27)$ & \\
\hline - Other & $2(29)$ & $4(27)$ & \\
\hline Child-Pugh score: & & & 0.6 \\
\hline - $\mathrm{A}$ & $2(29)$ & $6(40)$ & \\
\hline$\cdot \mathrm{B}$ & $2(29)$ & $1(7)$ & \\
\hline$\cdot \mathrm{C}$ & $3(43)$ & $8(53)$ & \\
\hline \multicolumn{4}{|l|}{ Preoperative laboratory } \\
\hline Prothombin time (sec) & $9.2(9-9.7)$ & $9.3(9-10)$ & 0.7 \\
\hline Bilirubine (mg/dl) & $1.5(0.95-2.25)$ & $2.7(1.3-5.35)$ & 0.4 \\
\hline AST (U/1) & $19(8-26.5)$ & $32(26-110)$ & 0.02 \\
\hline ALT (U/l) & $14(8.75-21)$ & $36(20-100)$ & 0.03 \\
\hline \multicolumn{4}{|l|}{ Perioperative characteristics } \\
\hline Cold ischemia time (min) & $225(185.5-232)$ & $198(136.5-268)$ & 0.8 \\
\hline Warm ischemia time (min) & $24(18.5-24)$ & $18(14-21)$ & 0.06 \\
\hline Anhepatic time (min) & $54(51.5-58)$ & $65(55-86.5)$ & 0.2 \\
\hline Surgery time (min) & $565(467.5-666.5)$ & $530(457.5-612.5)$ & 0.8 \\
\hline Graft-weight ratio (\%) & $1.08(0.94-1.38)$ & $0.9(0.82-1.11)$ & 0.23 \\
\hline
\end{tabular}



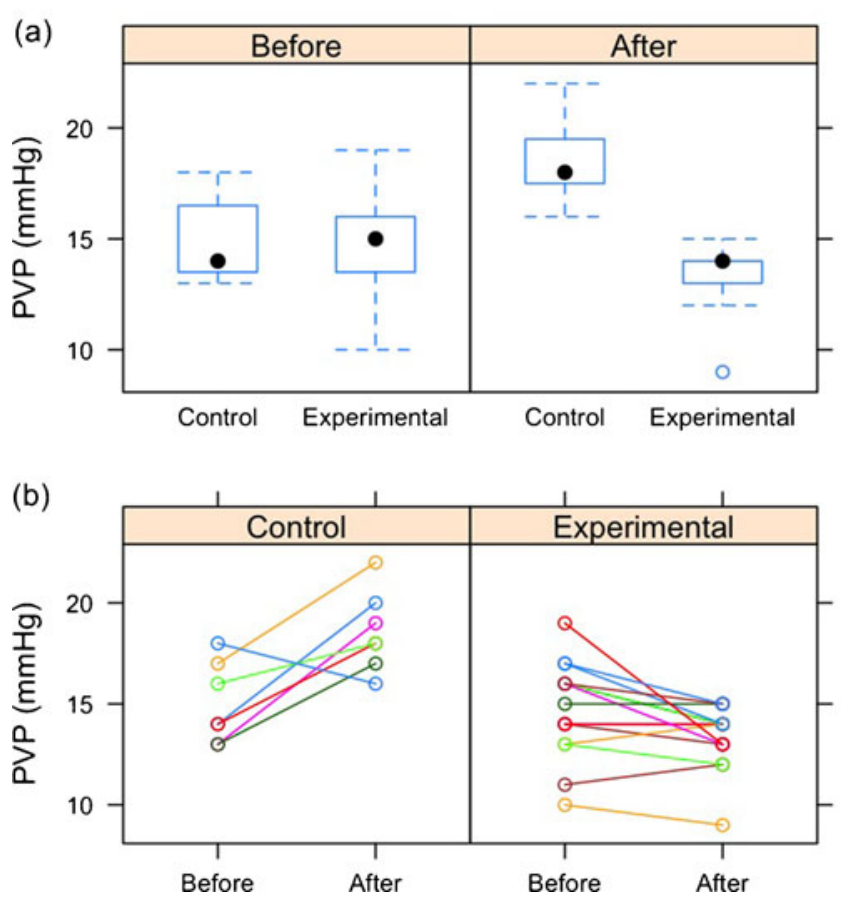

Fig. 4 Boxplot of portal vein pressure (PVP) before and after implantation of the graft (a). PVP before implantation is not different ( $p$ value $=0.98)$, whereas PVP after implantation of the graft is significantly lower in the experimental group ( $p$ value $<0.001)$. b Shows PVP before and after implantation of the graft within groups and for every single patient. Overall, PVP increases in the control group by $3.6 \mathrm{mmHg}$ ( $p$ value $<0.001$, CI $1.7-5.4)$, whereas there is a decrease by $4.9 \mathrm{mmHg}$ ( $p$ value $<0.001$, CI -7.2 to -2.6 ) in the experimental group

and ALT were also lower in the experimental group, but this did not reach statistical significance $(p$ value $=0.091$ and 0.078 for AST and ALT, respectively; Fig. 5). We did not observe differences for PT ( $p$ value 0.525 ) and GGT ( $p$ value $=0.237)$.

\section{Clinical outcome}

We did not observe primary nonfunction or acute rejection, but within the study period, one patient died with functioning graft due to infectious complications, one patient was re-transplanted on POD 5 in the control group due to severe graft congestion and one patient in the experimental group due to hepatic artery thrombosis with substantial graft necrosis on the 11th POD. We observed three SFSS in the control group (43\%, CI 12-80) and two SFSS in the experimental group $(13 \%$, CI $2-42)$; the difference being without statistical significance ( $p$-value $=$ 0.32 ). SFSS was treated by re-transplantation (one case) or symptomatic therapy (all other cases) and resolved in all patients after 3-6 weeks. We also did not observe significant differences in complications, in-hospital morbidity ( $p$ value $=0.60$ and 0.55 , respectively), and length of
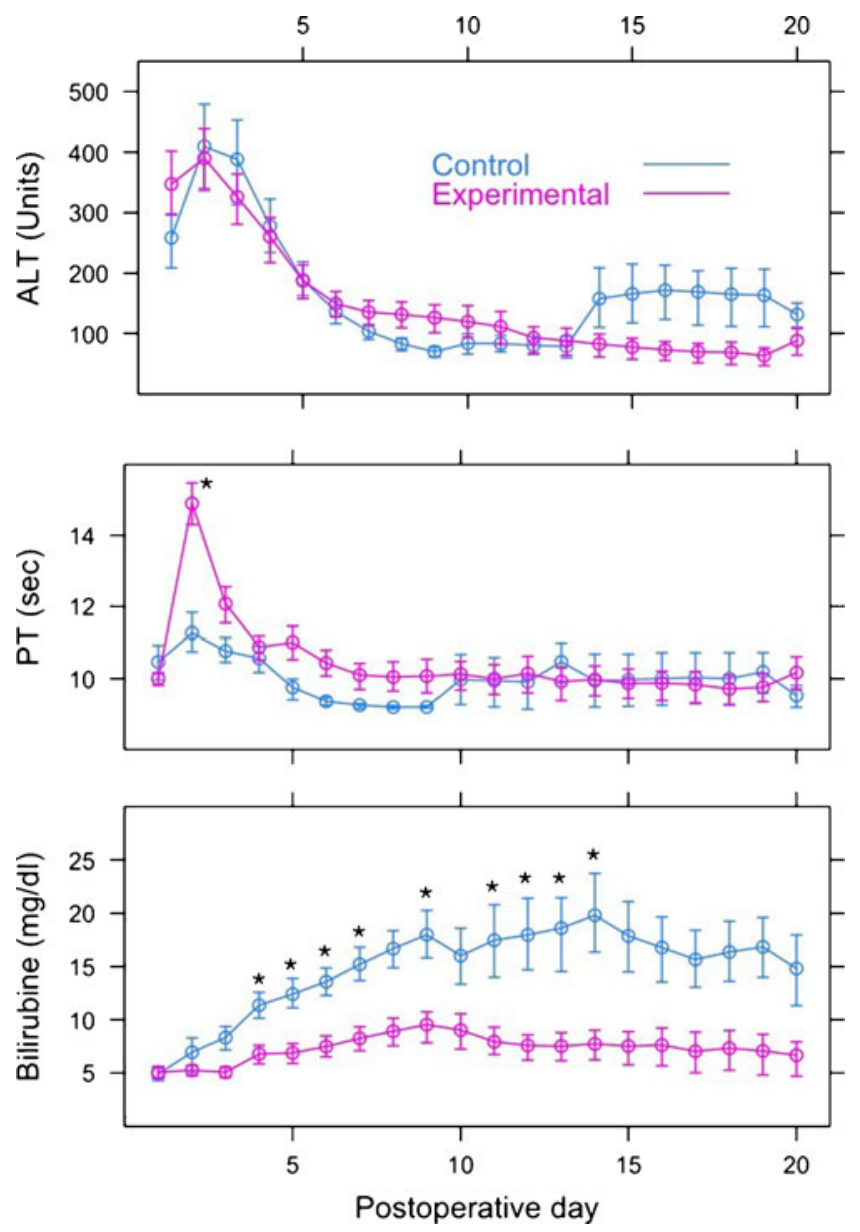

Fig. 5 Time course of laboratory measurements summarized within groups (mean and bootstrapped nonparametric 95\% confidence intervals for the estimate). $A L T$ alanine aminotransferase; $P T$ prothrombin time

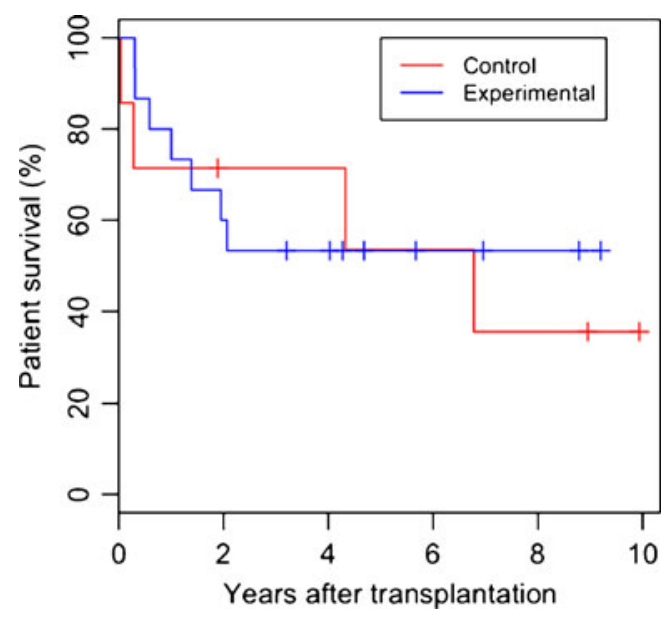

Fig. 6 Kaplan-Meier estimate of overall patient survival by study group. Log-rank test does not show significant differences between control group (red) and experimental group (blue, $p$ value $=0.76$ ) 
hospital stay (median 35 days, IQR 25-49). Kaplan-Meier estimates of overall patient survival (Fig. 6) are $77 \%$ at 1 year (CI 62-97), 58\% at 3 years (CI $40-83$ ), and $52 \%$ at 5 years (CI 34-80). Log-rank test does not show significant differences in patient or graft survival ( $p$ value $=0.76$ and 0.43 , respectively). In some patients, computed tomography (CT) was acquired for follow-up at 6 months or later, and these did not show significant stenosis at the anastomosis region in any of the patients of both groups.

\section{Discussion}

One of the key features of SFSS is hyperdynamic portal inflow and portal hypertension. Consequently, early attempts to avoid SFSS in LDLT aimed at portal decompression [1]. But more and more, it is recognized that portal decompression may also have deleterious effects on graft function [14] because liver regeneration is dependent on adequate graft inflow $[12,13]$. Fan and colleagues have also recognized the paramount importance on adequate outflow [1]. Outflow obstruction may not only aggravate inflow impedance but also increase portal hypertension, and thus mimic SFSS caused by hyperdynamic portal inflow and portal hypertension. Therapeutic strategies to divert portal inflow may backfire in this situation, because they will further reduce portal inflow and may cause a venous portal steal phenomenon [14]. Although many studies have shown beneficial effects of portal decompression [6-13], it has already been recognized that this strategy is not feasible in all patients receiving partial liver grafts [1, 14] and should be restricted to patients with increased portal inflow without outflow obstruction.

Conversely, Fan and colleagues have shown that optimizing outflow is one of the mainstays to avoid SFSS and should be attempted in all patients receiving partial liver grafts [1]. In addition to their elegant surgical technique of anterior cavoplasty, we propose a posterior cavoplasty, which should even be more effective in small grafts. With this new technique, we can show significant decrease of portal vein pressure, and this results in significantly less hyperbilirubinemia and lower rate of SFSS.

There has been much controversy concerning the management of the MHV and its tributaries [1]. In our center, it is a common policy to leave the MHV with the donor to minimize the donor risk but to reconstruct larger tributaries to the MHV. We decided to also reconstruct smaller tributaries in the control group, which may explain longer warm ischemia time compared to the experimental group. Multivariate analysis did not show any correlation between warm ischemia time and liver function tests, but weaker effects may not be excluded in this small cohort.
Small grafts should benefit from the described method of posterior cavoplasty because the small graft will tilt backwards in the large abdominal cavity (Fig. 2), and thus, a ridge will form at the posterior rim of the orifice of the RHV possibly even if the rim is incised as Fan proposes [1]. By means of posterior cavoplasty, the orifice of the RHV is enlarged in the dorsolateral direction, and we may prevent choking of the outflow tract by backwards tilt in the early phase after LDLT. Later on growth of the liver should adjust the functional anastomosis by rectifying the outflow tract. But regeneration is only possible when there is no small-for-size graft injury in the early phase. It might also be possible that in patients with posterior cavoplasty, a stenosis of the outflow tract will develop after growth of the liver, but we did not observe this in any of our patients.

In conclusion, we have shown that in this test-of-concept study, our new method of posterior cavoplasty resulted in significant reduction of PVP and bilirubinemia and a nonsignificant reduction of SFSS. Therefore, we recommend posterior cavoplasty to avoid venous outflow obstruction and possibly SFSS in small grafts. We are planning to confirm the findings of this feasibility study in a larger cohort of patients.

\section{Grants None.}

\section{Conflicts of interest None.}

Open Access This article is distributed under the terms of the Creative Commons Attribution Noncommercial License which permits any noncommercial use, distribution, and reproduction in any medium, provided the original author(s) and source are credited.

\section{References}

1. Fan ST (2007) Living donor liver transplantation. Takungpao Publishing, Hongkong

2. Emond JC, Renz JF, Ferrell LD, Rosenthal P, Lim RC, Roberts JP et al (1996) Functional analysis of grafts from living donors. Implications for the treatment of older recipients. Ann Surg 224 (4):544-554

3. Kiuchi T, Kasahara M, Uryuhara K, Inomata $\mathrm{Y}$, Uemoto S, Asonuma K et al (1999) Impact of graft size mismatching on graft prognosis in liver transplantation from living donors. Transplantation 67(2):321-327

4. Kiuchi T, Onishi Y, Nakamura T (2010) Small-for-size graft: not defined solely by being small for size. Liver Transplant 16:815-817

5. Man K, Fan ST, Lo CM, Liu CL, Fung PCW, Liang TB et al (2003) Graft injury in relation to graft size in right lobe live donor liver transplantation: a study of hepatic sinusoidal injury in correlation with portal hemodynamics and intragraft gene expression. Ann Surg 237(2):256-264

6. Lo CM, Liu CL, Fan ST (2003) Portal hyperperfusion injury as the cause of primary nonfunction in a small-for-size liver graft- 
successful treatment with splenic artery ligation. Liver Transpl 9 (6):626-628

7. Sato Y, Yamamoto S, Oya H, Nakatsuka H, Tsukahara A, Kobayashi $\mathrm{T}$ et al (2002) Splenectomy for reduction of excessive portal hypertension after adult living-related donor liver transplantation. Hepatogastroenterology 49(48):16521655

8. Ikegami T, Soejima Y, Taketomi A, Sanefuji K, Kayashima H, Harada $\mathrm{N}$ et al (2008) Living donor liver transplantation with extra-small graft; inflow modulation using splenectomy and temporary portocaval shunt. Hepatogastroenterology 55(8283):670-672

9. Boillot O, Delafosse B, Mechet I, Boucaud C, Pouyet M (2002) Small-for-size partial liver graft in an adult recipient; a new transplant technique. Lancet 359:406-407

10. Troisi R, Ricciardi S, Smeets P, Petrovic M, Van Maele G, Colle I et al (2005) Effects of hemi- portocaval shunts for inflow modulation on the outcome of small-for-size grafts in living donor liver transplantation. Am J Transplant 5(6):13971404

11. Troisi R, Cammu G, Militerno G, De Baerdemaeker L, Decruyenaere J, Hoste E et al (2003) Modulation of portal graft inflow: a necessity in adult living-donor liver transplantation? Ann Surg 237(3):429-436
12. Oya H, Sato Y, Yamamoto S, Takeishi T, Nakatsuka H, Kobayashi $T$ et al (2005) Surgical procedures for decompression of excessive shear stress in small-for-size living donor liver transplantationnew hepatic vein reconstruction. Transplant Proc 37(2):1108-1111

13. Taniguchi M, Shimamura T, Suzuki T, Yamashita K, Oura T, Watanabe $M$ et al (2007) Transient portacaval shunt for a smallfor-size graft in living donor liver transplantation. Liver Transpl 13(6):932-934

14. Boillot O, Pittau G, Bouffard Y, Sagnard P, Boucaud C (2009) Left liver lobe transplantation in adult recipients from split livers and living donors [abstract]. Transpl Int 22(Supp 2):61

15. Yu PF, Wu J, Zheng SS (2007) Management of the middle hepatic vein and its tributaries in right lobe living donor liver transplantation. Hepatobiliary Pancreat Dis Int 6(4):358-363

16. Agresti A (2002) Categorical data analysis. 2nd ed. John Wiley and Sons Inc.

17. Hollander M, Wolfe DA (1973) Nonparametric statistical methods. Wiley, New York

18. Pinheiro JC, Bates DM (2000) Mixed-effects models in S and SPLUS. Springer, Heidelberg

19. Hothorn T, Bretz F, Westfall P (2008) Simultaneous inference in general parametric models. Biom J 50(3):346-363

20. Efron B, Tibshirani R (1993) An introduction to the Bootstrap. Chapman \& Hall, London 